\title{
Correction to: WISP1 and TLR4 on Macrophages Contribute to Ventilator-Induced Lung Injury
}

\author{
Zhuang Yu, ${ }^{1}$ Tingting Wang, ${ }^{2}$ Liming Zhang, ${ }^{3}$ Xiaohu Yang, ${ }^{4,7}$ Quan Li, $^{5,7}$ and Xibing Ding ${ }^{6,7}$
}

Correction to: Inflammation (2020) 43(2):425-432

https://doi.org/10.1007/s10753-019-01103-0

In the published version of our article [1], we noticed an error in the "Funding" section. We inadvertently mentioned a grant from the National Institute of Health (R01GM-108639), which did not provide any support for the study. The statement should read, "This work was supported by the Jiangxi Natural Science Foundation Project (708174387032)."

Publisher's Note Springer Nature remains neutral with regard to jurisdictional claims in published maps and institutional affiliations.

The online version of the original article can be found at https://doi.org/ 10.1007/s10753-019-01103-0

\footnotetext{
${ }^{1}$ Department of Anesthesiology, Shanghai General Hospital, Jiaotong University School of Medicine, Shanghai, China

${ }^{2}$ Department of Anesthesiology, Huashan Hospital, Fudan University School of Medicine, Shanghai, China

${ }^{3}$ Department of Anesthesiology, University of Pittsburgh School of Medicine, Pittsburgh, PA, USA

${ }^{4}$ Department of Anesthesiology, Shanghai East Hospital, Shanghai, China

${ }^{5}$ Department of Anesthesiology, Cancer Hospital Chinese Academy of Medical Sciences, Shenzhen, China

${ }^{6}$ Department of Anesthesiology, Shanghai Renji Hospital, Jiaotong University School of Medicine, Shanghai, China

${ }^{7}$ To whom correspondence should be addressed to Xiaohu Yang at Department of Anesthesiology, Shanghai East Hospital, Shanghai, China. E-mail: shuaitiger@163.com; and to Quan Li at Department of Anesthesiology, Cancer Hospital Chinese Academy of Medical Sciences, Shenzhen, China. E-mail: quanligene@126.com; and ; 442546909@qq.com
} 